\title{
NONLINEAR ANALOGS OF LINEAR GROUP ACTIONS ON SPHERES
}

\author{
BY REINHARD SCHULTZ ${ }^{1}$
}

\section{Contents}

Introduction

1. Linear actions

2. Linear approximations to nonlinear actions

3. Analysis of actions with a given approximation

4. Fixed point sets

5. Homotopy linear actions: The basic setting

6. Homotopy linear actions: Some results and questions

7. Uniqueness of linear approximations

8. Final remarks

References

Introduction. This article is based upon a principle which is so standard that it is almost a cliché: The first step to understanding a nonlinear phenomenon is to define and study a suitable linear approximation. To be more specific, we shall describe some applications of this to the symmetry questions of topological transformation groups.

Given a topological space $X$, let $\operatorname{Homeo}(X)$ denote the set of self-homeomorphisms of $X$. This is a group under composition of mappings. If $G$ is an arbitrary group, then a group action of $G$ on $X$ is a homomorphism $\varphi$ : $G \rightarrow$ Homeo $(X)$. Frequently we wish to impose some weak assumptions on $\varphi$. For example, if $G$ is a topological group, then we might want $\varphi$ to have suitable continuity properties. The usual assumption is that the map

$$
\mu: G \times X \rightarrow X, \quad \mu(g, x)=\varphi(g)[x],
$$

is continuous; if $G$ has the discrete topology, then this condition is automatic. Also, it is often convenient to avoid homomorphisms that are in some sense degenerate. For example, every group maps into $\operatorname{Homeo}(X)$ by the constant homomorphism, but for many purposes this trivial sort of group action is uninteresting. The standard procedure is to limit attention to injective homomorphisms ( = effective group actions) unless stated otherwise.

Smooth actions. If $X$ is in fact a differentiable manifold with smooth structure (say) $\mathscr{F}$, it is often useful to consider group actions that are smooth in an appropriate sense. By this we mean that $G$ is a Lie group, $\varphi$ maps $G$ into the

Expanded version of an invited address presented at the Evanston, Illinois meeting in November 1983; received by the editors January 31, 1984.

1980 Mathematics Subject Classification. Primary 57S15, 57S17, 57S25; Secondary 55M35.

${ }^{1}$ Partially supported by NSF Grant MCS 83-00669. 
group $\operatorname{Diff}(X)$ of diffeomorphisms of $X$ (a subgroup of $\operatorname{Homeo}(X)$ ), and the map $\mu$ is smooth. The following classical result shows one context where smooth group actions arise in geometry:

THeOREM (COMPARE W. Y. HSIANG [48 P. 100]). Let $X$ be a compact smooth Riemannian manifold. Then the set of isometries $\operatorname{ISO}(X)$ is a Lie group, and the obvious homomorphism $\operatorname{ISO}(X) \rightarrow \operatorname{Homeo}(X)$ defines a smooth action.

Equivalent group actions. In virtually every mathematical context one needs a criterion for saying that two mathematical structures are essentially the same. For group actions the appropriate notion of (topological) equivalence is a homeomorphism $h \in \operatorname{Homeo}(X)$ such that

$$
\varphi^{\prime}(g)=h \varphi(g) h^{-1}, \quad \text { all } g \in G .
$$

This is equivalent to the relation $\varphi^{\prime}(g) h=h \varphi(g)$, and $h$ is often called an equivariant homeomorphism from $(X, \varphi)$ to $\left(X, \varphi^{\prime}\right)$.

Similar remarks apply to smooth actions, the main difference being that $h$ is taken to be in $\operatorname{Diff}(X)$.

1. Linear actions. The so-called linear actions on spheres form an important subclass of smooth actions. These actions correspond to continuous (equivalently, smooth) homomorphisms $\varphi$ from a compact Lie group $G$ to an orthogonal group $\mathrm{O}_{n}$; i.e. the actions correspond to orthogonal representations of $G$. Two representations $\varphi, \varphi^{\prime}$ are said to be equivalent if $\varphi^{\prime}=Q \varphi Q^{-1}$ for some $Q \in \mathrm{O}_{n}$. The linear actions on spheres then arise from the following elementary observations:

(1.1) If $S^{n-1}$ is the unit sphere in $\mathbf{R}^{n}$, then $\mathrm{O}_{n}$ maps $S^{n-1}$ to itself, and the induced homomorphism $\alpha: \mathrm{O}_{n} \rightarrow \operatorname{Homeo}\left(S^{n-1}\right)$ defines a smooth action. As a consequence, each representation $\varphi: G \rightarrow \mathrm{O}_{n}$ defines a smooth action of $G$ on $S^{n}$ via the composite $\alpha \varphi$.

Thus we have a transformation of mathematical theories of the following form:

$$
\begin{aligned}
& \text { \{orthogonal representations of } G \text { \} } \\
& \qquad L \downarrow \\
& \text { \{smooth actions of } G \text { on spheres }
\end{aligned}
$$

Results from representation theory give us a fairly good understanding of the representations of $G$ (compare W. Y. Hsiang's book [51]). Hence $L$ can be viewed as a mapping from a fairly well-understood theory to a less understood one. Of course, the linear actions are given by the image of $L$.

REMARK. A result of de Rham shows that $L$ is faithful; two linear actions are smoothly equivalent if and only if their representations are linearly equivalent [28] (also Rothenberg [84, Theorem 4.3, p. 300]). Incidentally, the corresponding statement is false if we replace "smoothly equivalent" by "topologically equivalent" (see Cappell-Shaneson [18]).

The discussion above motivates the following suggestion: To study NONLINEAR actions on spheres, first try to compare them with "linear approximations" of a suitable type. 
2. Linear approximations to nonlinear actions. The suggestion in the preceding paragraph assumes implicitly that one can define appropriate sorts of linear approximations. However, this is not obvious at first glance. Therefore we shall take some time to formulate this concept.

The following result shows that linear approximations to actions on $S^{1}$ and $S^{2}$ are trivial to construct:

THEOREM 2.0. Every continuous (resp., smooth) compact Lie group action on $S^{1}$ or $S^{2}$ is continuously (resp., smoothly) equivalent to a unique linear representation.

This fairly old result combines the work of several mathematicians, including H. Poincaré and L. E. J. Brouwer (see Edmonds [37]).

On the other hand, this sort of rigid behavior fails in higher dimensions. Nonlinear continuous actions exist on $S^{n}$ for every $n>3$ (compare Bing [6]), and nonlinear smooth actions exist on $S^{n}$ for every $n \geqslant 4$ (compare Giffen [42]; also see Gordon [43]); recent breakthroughs in 3-dimensional topology have shown that most (and perhaps all) smooth actions on $S^{3}$ are equivalent to linear actions (compare Morgan [66]). During the past three decades there have been many different constructions of nonlinear actions on $S^{n}$, and it is beyond the scope of this paper to survey these results fully. The survey article of Bredon [9] covers part of this material; the books of Bredon [12], Davis [24], Assadi [2] and W. Y. Hsiang [51] together with the articles of Petrie [74, 76, 80], Cappell-Shaneson [19], and the author [89-103, 108] provide further information on some of these questions.

How, then does one define a linear approximation to an arbitrary action on $S^{n}$ ? There are basically two possibilities:

(A) Define approximations using algebraic topology.

(B) Define approximations using geometric topology.

Our next order of business is to describe the mathematical substance behind these formal statements.

Algebraic-topological approximations. We shall illustrate the basic ideas when $G=\mathbf{Z}_{2}$. Let $T$ be the nontrivial element of $\mathbf{Z}_{2}$. If $\varphi$ is a $\mathbf{Z}_{2}$ action on $S^{n}$, let $\operatorname{Fix}(\varphi)$ be the action's fixed point set. If $\varphi$ is a linear action, then it is a routine exercise to verify that $\operatorname{Fix}(\varphi)=S^{k}$ for some $k<n$ and the degree $T$ is $(-1)^{n-k}$. A remarkable theorem of P. A. Smith yields a similar but weaker conclusion for arbitrary continuous actions. We shall state this result in a somewhat strengthened form (compare Bredon [12, Theorem III.7.11, p. 145]).

THEOREM 2.1 (essentially due to P. A. Smith). Let $\varphi$ be a $\mathbf{Z}_{2}$ action on $S^{n}$. Then $\operatorname{Fix}(\varphi)$ has the same Čech $\mathbf{Z}_{2}$ cohomology as $S^{k}$ for some $k<n$. Furthermore, degree $(T)=(-1)^{n-k}$.

Using this result it is trivial to construct a linear model for $\varphi$. If $I(m)$ represents the $m \times m$ identity matrix, then the linear approximation is defined by the $(n+1) \times(n+1)$ matrix

$$
B_{n-k}=\left(\begin{array}{cc}
I(k+1) & 0 \\
0 & -I(n-k)
\end{array}\right) .
$$

In other words, we take the representation $\varphi^{\prime}: \mathbf{Z}_{2} \rightarrow \mathrm{O}_{n+1}$ with $\varphi^{\prime}(T)=B_{n-k}$. 
There are some well-behaved classes of compact Lie groups to which one can extend these ideas at least partially. Versions of Smith's result are valid for actions of finite $p$-groups (where $p$ is any prime) and torus groups. This is best done using Borel's reformulation of Smith's results (the definitive reference is the Borel seminar book [8]).

Here is the basic idea. Let $\varphi$ be an action of $G$ on $S^{n}$, and let $G \rightarrow E G \rightarrow B G$ be the universal principal $G$-bundle. Then we may form the associated fiber bundle with fiber $S^{n}$ via

$$
E\left(S^{n}, \varphi\right)=S^{n} \times E G /(x, e) \sim(g x, g e) .
$$

We then have the following result (compare W. Y. Hsiang [49, Theorem 2, p. 280 ; or 51, pp. $72-75]$ ):

THeOREM 2.2. Let $G=\left(\mathbf{Z}_{p}\right)^{r}$ or $T^{r}$, let $\mathbf{F}=\mathbf{Z}_{p}$ or $\mathbf{Q}$, respectively, and suppose that $G$ acts on $S^{n}$. Let $S^{G}$ denote the action's fixed point set. Then the following hold:

(i) $H^{*}\left(S^{G} ; \mathbf{F}\right) \cong H^{*}\left(S^{k} ; \mathbf{F}\right)$ for some $k<n$.

(ii) There is a linear representation $\varphi^{*}$ on $\mathbf{R}^{n-k}$ such that the Serre spectral sequences for $H^{*}\left(E\left(S^{n}-S^{G}, \varphi\right) ; \mathbf{F}\right)$ and $H^{*}\left(E\left(S^{n-k-1}, \varphi^{*}\right) ; \mathbf{F}\right)$ are isomorphic.

If $\varphi^{*}$ denotes the representation in (ii), then $\varphi^{\prime}=\varphi^{*} \oplus$ trivial $(k+1)$ dimensional representation is an algebraically defined linear approximation to $\varphi$.

It is important to note that the representation $\varphi^{\prime}$ is usually NOT unique up to linear equivalence. For example, suppose $\alpha$ and $\beta$ are linear representations $G \rightarrow \mathrm{O}_{n+1}$ such that the fixed point sets of $\left(S^{n}, \alpha\right)$ and $\left(S^{n}, \beta\right)$ are empty and the bundles $E\left(S^{n}, \alpha\right), E\left(S^{n}, \beta\right)$ are fiber homotopy equivalent, i.e., there is a homotopy equivalence $h$ making the following diagram commutative:

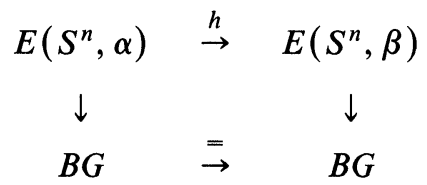

Then the cohomology Serre spectral sequences are automatically isomorphic. It is well known that such equivalences exist in many cases when $\alpha$ and $\beta$ are linearly inequivalent; the work of A. Meyerhoff and T. Petrie on quasiequivalence [62] provides one particularly large collection of examples.

In some cases it is possible to define linear approximations to more general actions than those of 2.2. For example, W. Y. Hsiang has studied this question in great detail if $G$ is a compact Lie group $[49,51]$; the key idea is to restrict the action of $G$ to the maximal torus. In another direction, it is sometimes possible to piece together a linear approximation to an action from linear approximations on subtori; this is considered in work of R. W. Sullivan [115, 116].

Geometric-topological approximations. We now assume that the compact Lie group $G$ acts smoothly on $S^{n}$. In this case we have the following fundamental 
result due to S. Bochner [7] (also see Bredon's book [12, Theorem VI. 2.2, pp. 306-307]):

THEOREM 2.3. Let $G$ act smoothly on a smooth manifold $M$, and let $x \in M$ be fixed under $G$. Then there is an open neighborhood $U$ of $x$ such that the following hold:

(i) $U$ is $G$-invariant, i.e., if $g \in G$ and $y \in U$ then $g y \in U$.

(ii) The induced smooth $G$-action on $U$ is smoothly equivalent to the action of $G$ on $\mathbf{R}^{m}$ by a well-defined linear representation $\rho(x): G \rightarrow \mathrm{O}_{n}$.

In fact, $\rho(x)$ is recoverable from the original action $\varphi$ as follows: Let $T_{x}$ $\left(\cong \mathbf{R}^{m}\right.$ ) be the tangent space at $x \in M$. Consider the map $\varphi^{\prime}: G \rightarrow L$ in Aut $\left(\mathbf{R}^{m}\right)$ which sends $g \in G$ to the derivative of $\varphi(g)$ at $x$. This is a continuous homomorphism by smoothness and the chain rule for differentiation, and $\rho(x)$ is merely an orthogonalization of $\varphi^{\prime}$.

It is a fairly routine exercise to show that the approximations given by Theorems 2.2 and 2.3 are compatible with each other. This is discussed in some detail in work of W. Y. Hsiang [49, 51] (loc. cit.).

For each point $x$ that is fixed under $G$, Theorem 2.3 yields a linear approximation to the original smooth action. In order to apply this observation effectively, we must be able to answer the following questions:

(i) If $\varphi$ is a linear action on $S^{n}$, how are its linear approximations related to the original representation?

(ii) How does the linear approximation $\rho(x)$ vary with respect to the choice of fixed point $x$ ?

The answer to the first question is basically a routine consequence of the definitions:

THEOREM 2.4. Let $\varphi_{0}$ be a linear representation on $\mathbf{R}^{n+1}$, and let $\varphi$ be the associated linear action on $S^{n}$. Assume that $\varphi$ has a nonempty fixed point set. Then $\varphi_{0}$ splits as a direct sum $\varphi_{1} \oplus \mathbf{R}$ (trivial representation on the second factor) and, for each fixed point $x$, the representation $\rho(x)$ is equivalent to $\varphi_{1}$.

In particular, $\rho(x)$ and $\varphi_{0}$ are equivalent up to addition of a trivial factor.

General considerations of transformation groups also say a great deal about question (ii).

THEOREM 2.5. Let $\varphi$ be a smooth action of $G$ on $S^{n}$ with nonempty fixed point set $F$. If $x$ and $y$ lie in the same connected component of $F$, then the representations $\rho(x)$ and $\rho(y)$ are equivalent.

In fact, Bochner's theorem implies that the equivalence class of $\rho(x)$ is locally constant; the class of $\rho(x)$ is constant for linear actions, and the given action $\varphi$ is linear near $x$.

COROLlARY 2.6. (i) If $F$ is connected, then there is a unique linear approximation up to equivalence.

(ii) An arbitrary smooth action with $F \neq \varnothing$ defines at most finitely many linear approximations up to equivalence. 
Part (ii) follows because $F$ is a closed locally connected subset of $S^{n}$ and therefore has finitely many components.

Problem 2.7. Which finite lists of representations $\left\{\rho_{j}: G \rightarrow \mathrm{O}_{n}\right\}$ occur as linear approximation to a smooth action on $S^{n}$ ?

This is a fairly basic question, and we shall discuss it further in $§ 7$. Since we shall be discussing actions of $\mathbf{Z}_{p}$ ( $p$ prime) in greater detail, at this time we shall only describe what happens for $G=\mathbf{Z}_{p}$. The following result is a consequence of the P. A. Smith theorems and a theorem of Atiyah and Bott [4, $\S 7$, pp. 474-479].

THEOREM 2.8. A smooth action of $\mathbf{Z}_{p}$ on $S^{n}$ with $F \neq \varnothing$ has a unique linear approximation.

Steps In the Proof. The P. A. Smith fixed point theorems imply that $F$ is a $\bmod p$ cohomology sphere [12, p. 145]. Hence $F$ is either connected or has the cohomology of $S^{0}$. Since $F$ is a union of orientable submanifolds, the latter implies that $F=S^{0}$. However, if the fixed point set is $S^{0}$ one can apply the fixed point formula of Atiyah and Bott [7], and by [7, \$7] one knows that the representations at the two fixed points are equivalent.

The discussion above assumes explicitly that the action has at least one fixed point. If the action $\varphi$ has no fixed points, it is sometimes still possible to construct a linear approximation or something that looks very much like one. Suppose there is a family $\left\{H_{j}\right\}$ of closed subgroups of $G$ with the following properties:

(i) The conjugates of the $H_{j}$ generate $G$.

(ii) For each $H_{j}$, the fixed point set of $H_{j}$ is nonempty.

These conditions yield representations $\rho_{j}$ of $H_{h}$, and frequently there is at most one representation $\rho$ with $\rho \mid H_{j}=\rho_{j}$. The problem is to find this representation if it exists. Even if such a representation $\rho$ does not exist, it may be possible to define a "generalized representation" by some formal device; for example, one might obtain a rational linear combination of real representations.

EXAMPLES. $1 . G$ is connected, $H$ is a maximal torus, and the sphere is even dimensional (see [12, Chapter II], for a proof that $H$ has a nonempty fixed point set). This case was studied extensively by W. Y. Hsiang (e.g., [49, 51]).

2. $G$ is an $r$-torus, $\left\{H_{j}\right\}$ is a judiciously chosen finite family of $(r-1)$-subtori. Even if $G$ acts without fixed points, cohomological methods imply that finitely many $(r-1)$-subtori have nonempty fixed point sets (compare W. Y. Hsiang [49, p. 276]). The objective is then to fit the representations $\rho_{j}$ together to obtain a representation of $G$ or something close. This problem was studied by R. W. Sullivan $[115,116]$.

3. Suppose $G=\mathrm{SO}_{3}$ and take the family of subgroups $\left\{\mathrm{O}_{2}\right.$, symmetry group of the standard cube $\}$. This case was studied in great detail by R. Oliver [69] for actions on disks.

FINAL REMARK. There are also many further results on linear approximations for $G$-actions on $D^{n}$ or $\mathbf{R}^{n}$ without fixed points (compare W. Y. Hsiang $[49,51])$. 
We may summarize the discussion of this section as follows:

(2.9) Bochner's theorem defines a linear approximation to a smooth G-action on $S^{n}$ with the following properties:

(i) The fixed point set is nonempty.

(ii) For every fixed point $x$, the representation $\rho(x)$ is equivalent to some fixed representation $\rho$.

If $p$ is prime, then (ii) holds for all actions of $\mathbf{Z}_{p}$.

3. Analysis of actions with a given approximation. Suppose now that we are given a smooth $G$-action $\varphi$ on $S^{n}$ with linear approximation on $\varphi^{\prime}$. It is natural to consider the similarities and differences between $\varphi$ and $\varphi^{\prime}$. During the past two decades the methods of algebraic and geometric topology have provided a great deal of insight into two phases of analyzing $\varphi$ with respect to $\varphi^{\prime}$ :

(I) Comparison of the fixed point set of $\varphi$ with that of $\varphi^{\prime}$.

(II) Description of all actions with the same fixed point sets as in the linear approximation.

If $G=\mathbf{Z}_{p}$ ( $p$ prime), then (I) and (II) yield a fairly complete analysis of all actions with a fixed linear approximation; this principle is reflected in work of L. Jones $[53,54]$ and the author $[93,99,101]$. We shall describe the answers to (I) and (II) for $G=\mathbf{Z}_{p}$ and comment on their generalizations to actions of more general groups.

In order to keep the discussion relatively concise and unified, we henceforth concentrate on actions of finite groups. Some of the results extend to actions of $S^{1}, S^{3}$, and torus groups (see the papers of Browder and Petrie [17] and the author [88-91, 94, 104-107]).

If the group $G$ acting on $S^{n}$ is relatively large (e.g., $\operatorname{dim} G>2 n$ ), then it is once again possible to analyze actions with a fixed linear approximation in great detail. However, the methods are somewhat different from those discussed here. Details may be found in papers of the Hsiangs (e.g., [46-48]) and M. Davis ([24 and 25] give an excellent overview).

4. Fixed point sets. In this section we wish to consider the following question: What are the possible fixed point sets of a smooth $\mathbf{Z}_{p}$ action on $S^{n}$ ?

Before we do this, we recall some fundamental observations.

(4.1) Suppose $G$ (compact Lie) acts linearly on $S^{n}$, and let $H \subseteq G$ be a closed subgroup. Then the H-fixed point set

$$
\operatorname{Fix}\left(H, S^{n}\right)=\left\{x \in S^{n} \mid h x=x, \text { all } h \in H\right\}
$$

is a standardly embedded subsphere. If $H$ is normal in $G$, then $\operatorname{Fix}\left(H, S^{n}\right)$ is a G-invariant smooth submanifold.

This follows because if $G$ acts linearly on $\mathbf{R}^{n+1}$, then the set of vectors fixed under $H$ is a vector subspace.

TheOREM 4.2 (Equivariant Tubular Neighborhood Theorem; see Bredon [12, Theorem VI. 2.2, pp. 306-307]). Let $M^{m}$ be an unbounded smooth G-manifold, $N$ a closed unbounded invariant smooth submanifold. Then $N$ has an invariant 
tubular neighborhood, i.e., a G-invariant open neighborhood $V \supseteq N$ such that the following hold:

(i) $V$ is equivalent to the total space of a vector bundle over $N$ (i.e., a smoothly parameterized family of vector spaces over $N$ with points of $N$ corresponding to the zero vectors).

(ii) Each $g \in G$ maps the vector space $V_{x}$ over $x \in N$ linearly to the vector space $V_{g x}$ over gx (see Figure 1).

The vector bundle over $N$ in 4.2 is called the equivariant normal bundle of $N$ in $M$. It is unique up to an appropriate notion of $G$-equivariant1 vector bundle isomorphism (Bredon [12, Theorem VI. 2.6, pp. 310-311]).

OBSERVATION 4.3. Both (4.1) and 4.2 apply to the fixed point set of a linear $G$-action on $S^{n}$. In this case the equivariant normal bundle is isomorphic to the trivial bundle $\pi: S^{k} \times\left(\mathbf{R}^{n-k}, \psi\right) \rightarrow S^{k}(\pi(x, v)=x)$, where $\psi$ is some linear representation of $G$ with zero-dimensional fixed point set.

The proof of the second sentence in 4.3 is elementary, but not written down explicitly in a prominent place, so we do so here. If the linear action $\varphi$ on $S^{n}$ has a $k$-dimensional sphere as its fixed point set, then the underlying representation $\varphi_{0}$ on $\mathbf{R}^{n+1}$ splits as a product $\mathbf{R}^{k+1} \times\left(\mathbf{R}^{n-k}, \psi\right)$, where $\varphi$ is as described in 4.3. Define a map $h: S^{k} \times D^{n-k} \rightarrow S^{n}$ via

$$
h(x, v)=\left(\sqrt{1-|v|^{2} / 4} x, v / 2\right) \quad\left(\in \text { unit sphere in } \mathbf{R}^{k+1} \times \mathbf{R}^{n-k}\right)
$$

(see Figure 2).

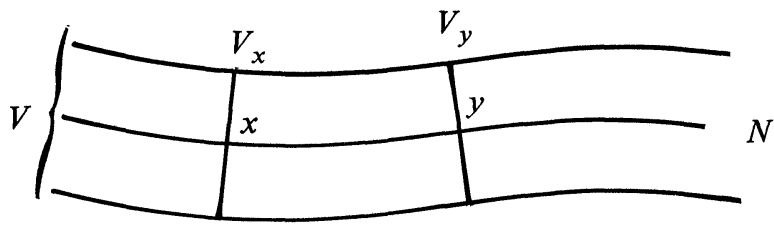

FIGURE 1

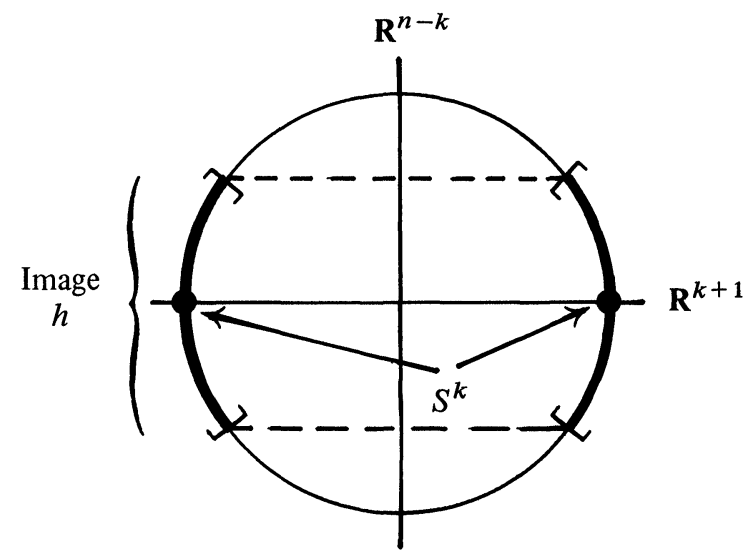

FIGURE 2 
Let $k: \mathbf{R}^{n} \rightarrow$ Int $D^{n}$ be the diffeomorphism

$$
k(y)=y /(1+|y|),
$$

and set $h^{*}(x, y)=h(x, k(y))$. Then $h^{*}$ defines a diffeomorphism from $S^{k} \times$ $\mathbf{R}^{n-k}$ onto an invariant neighborhood of $S^{k}$. Under $h^{*}$ the $G$-action corresponds to the action on $S^{k} \times \mathbf{R}^{n-k}$ via

$$
g(x, y)=(x,[\psi(g)](y))
$$

this follows directly from the linear splitting of $\mathbf{R}^{n+1}$ as described above.

We now return to the central question on fixed point sets of smooth $\mathbf{Z}_{p}$ actions on $S^{n}$. The following are necessary conditions on the fixed point set $F$ of such an action:

(4.4) $F$ is a smoothly embedded, closed, oriented submanifold.

This follows by general principles [12, Theorem V. 2.1, p. 175].

(4.5) $F$ has the mod $p$ cohomology of $S^{k}$ for some $k$ with $k<n$. If $p$ is odd, then $n-k$ is even. If $p=2$, then $(-1)^{n-k}=\operatorname{degree} T$, where $1 \neq T \in \mathbf{Z}_{2}$.

This follows from Smith theory [12, p. 145].

(4.6) If $p$ is odd, the normal bundle of $F$ in $S^{n}$ splits into a sum of $(p-1) / 2$ complex vector bundles.

This follows from the elementary theory of $G$-vector bundles (compare Segal [110, Proposition 2.2, p. 133]). Intuitively, it may be seen as follows. If $A$ is a $q \times q$ matrix with $A^{p}=I$ and 1 is not an eigenvalue, then $A$ splits $\mathbf{R}^{q}$ into $(p-1) / 2 A$-invariant subspaces, each of which has a complex structure; some of the subspaces may be zero dimensional. (4.6) is merely a parameterized version of this.

If $k=\operatorname{dim} F$ is even, the complex vector bundles in (4.6) all have rational Chern classes; these lie in $H^{k}(F, \mathbf{Q})=\mathbf{Q}$ (see Milnor and Stasheff [64]). These characteristic classes can be studied via the Atiyah Singer $G$-signature formula $[5, \S 6]$ and some number theoretic computations. The following result of J. Ewing [39] provides an important condition on these classes.

(4.7) If $\operatorname{dim} F \neq 2$, then the rational Chern classes of the bundles in (4.6) are all trivial.

Note. In the linear case this is immediate because the vector bundles in (4.6) are always trivial bundles.

The restriction $\operatorname{dim} F \neq 2$ is curious but need not concern us here; if $\operatorname{dim} F=2$ and $F$ is an oriented $\bmod p$ homology sphere, then $F$ must be diffeomorphic to $S^{2}$ by the classification theory of oriented surfaces (e.g., see Hirsch [44, Chapter 9]).

Conditions (4.4)-(4.7) are in fact very close to being sufficient for realizing $F^{k}$ as a fixed point set. For the sake of simplicity we restrict attention to the case where $n>2 k$ (i.e., the general position on gap hypothesis range). Forthcoming papers of A. Assadi and W. Browder [3] and S. Cappell and S. Weinberger [20] shed considerable light on the case $n \leqslant 2 k$.

If $n>2 k$, then by the Whitney embedding theorem $F$ embeds smoothly in $S^{n}$. This smooth embedding has a normal bundle that we shall call $\nu$. We use this notation in stating the following converse to (4.4)-(4.7). The initial results and key insights were due to L. Jones [53, 54], with subsequent contributions 
by a number of others (see the bibliography to [101]; also, see the paper of Cappell and Weinberger).

THEOREM 4.8. Suppose $F^{k}$ is a closed oriented mod $p$ homology sphere, and suppose that the normal bundle $\nu$ satisfies (4.6) and (4.7). Then there is a smooth $\mathbf{Z}_{p}$ action on $S^{n}$ whose fixed point set $E$ is almost diffeomorphic to $F$.

By "almost" we mean that $E-\{\mathrm{pt}\}$ is diffeomorphic to $F-\{\mathrm{pt}\}$; it follows that $E$ and $F$ are homeomorphic by taking one-point compactifications.

The "almost diffeomorphism" condition is very curious. If $p$ is odd and $n<k p$, by results of P. Löffler [60] one can find actions where $E$ and $F$ are diffeomorphic (compare [101, Theorem B]). On the other hand, there is a sequence of integers $M_{k}$ such that "almost diffeomorphism" is indispensable for $n \geqslant M_{k}$ (compare [101, Theorem C]). These results are closely tied to two important global theorems in stable homotopy theory-the Kahn-Priddy Theorem [55] and the solution of the Segal Conjecture [58, 59] (see [1] for an overview).

Actions of more general groups. In linear representation theory one begins by describing basic properties of representations of finite cyclic groups. Once these properties seem relatively well understood, the next step is to study the corresponding questions for representations of increasingly wider classes of groups. The analogous approach is frequently meaningful and appropriate for nonlinear actions on spheres. For example, the P. A. Smith theorems on $\mathbf{Z}_{p}$ actions have numerous extensions to actions of finite p-groups (compare Bredon [12]). This suggests one general pattern of inquiry:

(4.9) Given a result on $\mathbf{Z}_{p}$ actions, to what extent does it extend to actions of a broader class of finite groups?

In view of Theorem 4.8 we have a natural test case-namely, the description of fixed point sets of smooth $G$-actions on $S^{n}$. We split the discussion into three parts.

1. Semifree actions. A $G$-action is semifree if each $x$ is either (a) fixed under all of $G$, (b) not mapped to itself by any element of $G$ except the identity. Since $G_{x}=\{g \in G \mid g x=x\}$ is a subgroup, it follows that every $\mathbf{Z}_{p}$-action is semifree (there are only the two obvious subgroups). A group $G$ acts semifreely with nonempty fixed point set if and only if it admits a free linear representation ( $g v=v$ and $v \neq 0$ imply $g=1$ ). Therefore the set of all such groups consists of closed subgroups of $S^{3}$ and the classical spherical space form groups as described in Wolf [122] (see Bredon [12, §III.8] for a discussion of the case $\operatorname{dim} G>0$ ).

If $G=S^{1}$, the normalizer of $S^{1}$ in $S^{3}$, or $S^{3}$ itself, there is an extremely good generalization of 4.8 to semifree $G$-actions. In these cases one knows that the fixed point set $F^{k}$ must be an integral homology sphere (compare Bredon [12, Corollary III.10.14, p. 166]).

The following result actually preceded Theorem 4.8 historically:

Theorem 4.10 (HSIANg AND HSIANg [46]). Let $F$ be a closed smooth Z-homology sphere, assume $\operatorname{dim} F \neq 3$, and let $d=2$ if $G=S^{1}$ and $d=4$ otherwise. Then for all $m \geqslant 1$ there is a smooth semifree G-action on $S^{k+n d}$ with fixed point set $E$ almost diffeomorphic to $F$. 
If $G$ is finite there is again a decent generalization of Theorem 4.8 to semifree $G$-actions (e.g., see Assadi [2, Theorem VI 2.4, p. 101]). However, there is one extra twist. By Smith theory the fixed point set must be a $\mathbf{Z}_{p}$ homology sphere for all primes $p$ dividing the order of $G$. Therefore the groups $H_{i}=H_{i}(F ; \mathbf{Z}), 0<i<k$, must be torsion of order prime to $|G|=\operatorname{order} G$. Assume that $G$ acts trivially on $H_{i}$ and view $H_{i}$ as a $\mathrm{Z}[G]$ module in this way. Classical results in homological algebra imply that the $H_{i}$ have short projective resolutions $0 \rightarrow P_{1}(i) \rightarrow P_{0}(i) \rightarrow H_{i} \rightarrow 0$ with $P_{0}(i)$ free (compare Rim [82, $\S 4])$. Therefore one can define an Euler characteristic

$$
\boldsymbol{\sigma}(F)=-\sum(-1)^{i}\left[P_{1}(i)\right] \in \tilde{K}_{0}(\mathbf{Z}[G]),
$$

where $K_{0}(\mathbf{Z}[G])$ denotes the reduced projective class group (see Milnor [63]); the minus sign arises because $P_{1}(i)$ and $H_{i}$ are inverses in some appropriate Grothendieck group. This type of invariant arose in work of Swan during the 1950s [117]. The significance of $\sigma(F)$ is given by the following result:

(4.11) If $F$ is the fixed point set of a semifree $G$-action on $S^{n}$, then $\sigma(F)=0$.

See Assadi [2A] for further details. Conversely (compare [2, 2A]), if $F$ satisfies (4.11) and the natural analogs of (4.4)-(4.7), then $F$ is almost diffeomorphic to the fixed point set of a semifree $G$-action.

2. Actions of finite p-groups. In this case P. A. Smith theory provides strong restrictions ([12] again) on possible fixed point sets and their homological properties. However, at this time there are very few realization theorems like (4.8) except for semifree actions of $\mathbf{Z}_{p^{r}}, r \geqslant 2$. Recently S. Weinberger has developed an alternate approach to the results of L. Jones (see Weinberger [121]). It appears likely that Weinberger's methods will lead to considerable progress in this study of fixed point sets of $p$-group actions.

3. Actions of non-p-groups. In contrast to cases 1 and 2 , there are no theorems of P. A. Smith type to restrict the fixed point structure. The failure of Smith theory for $G=\mathbf{Z}_{6}$ was already noted by E. E. Floyd in the 1950s [40]. Results of Oliver [69] provide an excellent basis for understanding the structure of fixed point sets for non-p-group actions. The work of Assadi [2] contains numerous further developments and studies the fixed point sets of actions on spheres in considerable detail. For example, we have the following results:

TheOREM 4.12 (ASSADI [2, Theorem IV. 5.1, p. 87]). Let $P$ be a finite polyhedron, and let $G$ be a finite group that is "sufficiently large" in the sense of a complicated lattice of subgroups (see [2, p. 87] for specifics). Then there is an $n>0$ and a simplicial action $\varphi$ of $G$ on some subdivision of (the standard triangulation of ) $S^{n}$ with $P$ as the fixed point subcomplex.

THEOREM 4.13 (specialized from [2A, Corollary IV.3.1, pp. 84-85]). ${ }^{2}$ Let $P=\partial Q$, where $Q$ is a parallelizable manifold of odd Euler characteristic, and let $G$ be as above. Then there is an $n>0$ and a smooth action $\varphi$ of $G$ on $S^{n}$ with $P$ as the fixed point set.

\footnotetext{
${ }^{2}$ Combine that result with Example 1.4.2, pp. 23-34, and the definition of $\Phi$ on p. 87.
} 
In general, the realization question for fixed point sets of a $G$-action is very deep and difficult. This is true even if one considers specific, simple examples such as $F=\{\mathrm{pt}\}$. For example, the following striking result due to T. Petrie [77] requires an extremely large amount of technical preparation and a very broad range of ideas:

TheOREM 4.14. (PETRIE [77]). Let $G$ be a "sufficiently large" odd order abelian group (i.e., at least three noncyclic Sylow subgroups). Then there is a smooth action of $G$ on some $S^{n}$ with exactly one fixed point.

It is important to note that the work of Oliver [69] yields a converse to Petrie's theorem:

(4.15) If $G$ is finite abelian and acts smoothly on $S^{n}$ with one fixed point, then $G$ has at least three noncyclic Sylow subgroups.

There has been a great deal of progress on understanding fixed point sets of non-p-group actions in the past dozen years. However, very much remains to be learned.

5. Homotopy linear actions: The basic setting. The viewpoint of this section is almost completely opposite to that of the previous section. We wish to describe all actions whose fixed point structure agrees with that of the linear approximation. As noted in $\S 3$, if $G=\mathbf{Z}_{p}$ then the viewpoints of $\S 4$ and this section provide a fairly comprehensive description of all possible $\mathbf{Z}_{p}$ actions.

The terms "homotopy linear" and "semilinear" first appear explicitly in papers of tom Dieck [29, 30] and tom Dieck and Petrie [31]. However, the concept dates back to the 1960s and some results of Bredon [9, §6] and Montgomery-Yang [65] (also see [103]); shortly afterwards, Browder and Petrie [17] and Rothenberg and Sondow [85] (also see [83]) began a systematic study of actions that were semilinear and semifree.

Formal definition. Let $\varphi_{0}$ be a linear representation of $G$ on $\mathbf{R}^{n+1}$. If $H$ is a subgroup of $G$, then $\operatorname{Fix}\left(H, \mathbf{R}^{n+1}\right)$ is a vector subspace; denote its dimension by $n(H)+1$. Assume $n(H) \geqslant 0$. A smooth $G$-action $\varphi$ on a smooth manifold $\Sigma^{n}$ is said to be $\varphi$-homotopy linear (or semilinear) if the following hold:

(i) For each $H \subseteq G$ the fixed point set of $H$ is homeomorphic (sic) to $S^{n(H)}$.

(ii) If $H \subseteq K \subseteq G$ and $n(H)-n(K)=2$, then $\operatorname{Fix}(H, \Sigma)-\operatorname{Fix}(K, \Sigma)$ is homotopy equivalent to $S^{1}$.

The second assumption is often irrelevant, but it does allow us to avoid any possible problems with knot theory.

The following result explains the term "homotopy linear".

Proposition 5.1. Let $\Sigma^{n}$ be a $\varphi$-homotopy linear sphere, and assume that $x \in \Sigma$ is a fixed point with linear approximation satisfying $\rho(x) \oplus \mathbf{R} \cong \varphi($ as representations). Then $\Sigma^{n}$ is G-equivariantly homotopy equivalent to the linear action $\left(S^{n}, \varphi\right)$.

REMARKs. In fact, $\Sigma^{n}$ and $\left(S^{n}, \varphi\right)$ are often $G$-topologically equivalent (compare Connell-Montgomery-Yang [22], Illman [52]).

Proof. By the assumption and Bochner's theorem, $x$ has a neighborhood $U$ on which $\varphi$ acts linearly. Map $\Sigma^{n}$ to the one-point compactification $U^{*}$ by 
collapsing $\Sigma-U$ to a point; this is a $G$-equivariant map. Notice that $U^{\cdot}$ is $G$-equivalent to $\left(S^{n}, \varphi\right)$, and hence we have a map $f: \Sigma^{n} \rightarrow\left(S^{n}, \varphi\right)$. It is immediate from the construction that, for each subgroup $H$, the induced map of $H$-fixed points sets,

$$
\operatorname{Fix}(f ; H): \operatorname{Fix}\left(\Sigma^{n} ; H\right) \rightarrow \operatorname{Fix}\left(S^{n} ; H\right),
$$

has degree 1; in other words, each map $\operatorname{Fix}(f ; H)$ is a homotopy equivalence. Therefore $f$ is an equivariant homotopy equivalence by an equivariant generalization of Whitehead's Theorem (e.g., see Matumoto [61]).

Connected sums. The $\varphi$-semilinear actions may be viewed as equivariant generalizations of exotic spheres; i.e., manifolds $\Sigma^{n}$ that are homeomorphic but not diffeomorphic to $S^{n}$. During the late 1950s and early 1960s M. Kervaire and J. Milnor developed a classification theory for such manifolds [56]; this work has had very far-reaching implications for geometric topology. Therefore it seems reasonable to extend as much of the Kervaire-Milnor classification as possible to study $\varphi$-homotopy linear $n$-manifolds.

The most elementary step in the Kervaire-Milnor program is to define a group operation on diffeomorphism classes of exotic spheres. This is done using the notion of connected sum (compare Bröcker and Jänich [15, Chapter 10]). Given two disjoint exotic spheres $S_{1}$ and $S_{2}$, one takes closed disks $D_{i} \subseteq S_{i}$ and joins $S_{1}-$ Int $D_{1}$ to $S_{2}-$ Int $D_{2}$ by a tube $T \cong S^{n-1} \times[0,1]$ (see Figure 3).

This description is slightly inaccurate because we must be careful about orientations; both $S_{1}$ and $S_{2}$ are assumed to be oriented, and the diffeomorphisms $f_{i}: D^{n} \rightarrow D_{i}$ must be chosen so that one preserves orientation and one reverses orientation. Without these precautions the connected sum is not well defined.

One can proceed similarly with homotopy linear $\varphi$-spheres. In this context the disks $D_{i}$ are taken to be $\varphi$-linear disks centered at fixed points of the action. Once again it is necessary to be careful about orientations; in fact, one needs a suitable concept of equivariant orientation which is slightly tricky but not

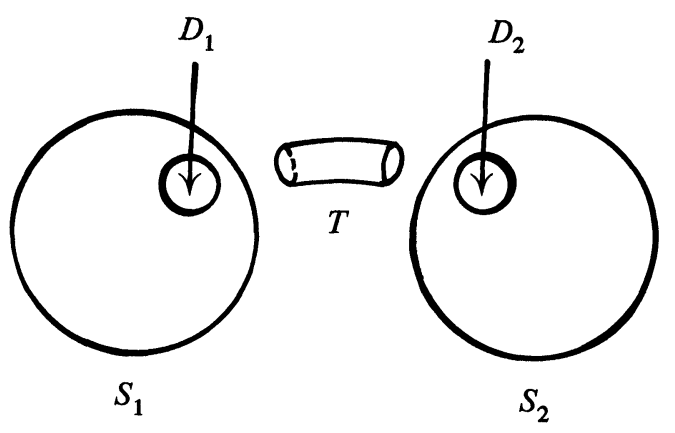

FIGURE 3 
really deep (compare $[94, \S 1])$. The main conclusion can then be stated as follows:

TheOREM 5.2. Let $\varphi$ be a linear representation of $G$ on $\mathbf{R}^{n+1}$ with $k=$ $\operatorname{dim} \operatorname{Fix}\left(G, \mathbf{R}^{n+1}\right)>0$.

(i) The set $H L(\varphi)$ of $G$-oriented equivalence classes of semilinear $\varphi$-spheres is a monoid with respect to equivariant connected sum. If $k \geqslant 2$ this monoid is abelian.

(ii) Let $K L(\varphi)$ be the submonoid of semilinear $\varphi$-spheres that bound equivariantly contractible manifolds. Then the quotient monoid

is a group.

$$
\Theta^{G}(\varphi)=H L(\varphi) / K L(\varphi)
$$

In fact, one can describe the inverse to a homotopy linear $\varphi$-sphere $\Sigma^{n}$ very explicitly; it is just $\Sigma^{n}$ equipped with the negative of the original equivariant orientation.

If $G=1$ these groups are the same as the Kervaire-Milnor groups $\Theta_{n}$ provided $n \neq 3$; this follows from the Generalized Poincaré Conjecture (compare Kervaire and Milnor [56, Remark, p. 505]; also see Freedman [41]).

ADDED IN PROOF. The results of [29-31] on homotopy linear actions also provide a setting for studying linear approximations to nonlinear actions from a viewpoint that differs somewhat from those of $\S 2$. Further details will appear in a forthcoming book by tom Dieck and related work of I. Madsen and M. Raussen.

6. Homotopy linear actions: Some results and questions. Given that the groups $\Theta^{G}(\varphi)$ are generalizations of the Kervaire-Milnor groups $\Theta_{n}$, it is reasonable to analyze them using similar methods. Therefore it is appropriate to recall two major points in the Kervaire-Milnor program; we shall do so in slightly different terms that are equivalent to those of [56] (compare Levine [57]).

Let $F$ be the limit of the sequence

$$
\cdots \rightarrow \operatorname{Cont}\left(S^{n}, S^{n}\right) \stackrel{\Sigma}{\rightarrow} \operatorname{Cont}\left(S^{n+1}, S^{n+1}\right) \rightarrow \cdots,
$$

where Cont( , ) is the space of continuous maps (say with the compact-open topology) and $\Sigma$ is the suspension map. There is a natural inclusion $J_{n}$ of $\mathrm{O}_{n+1}$ in $\operatorname{Cont}\left(S^{n}, S^{n}\right)$, and one can form a topological quotient $(F / \mathrm{O})_{n}$ of the monoid $\operatorname{Cont}\left(S^{n}, S^{n}\right)$ by the subgroup $\mathrm{O}_{n+1}$. From this one obtains the following sort of commutative diagram:

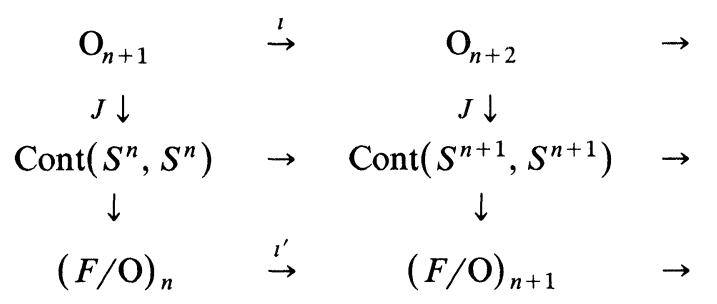

Thus we can form $F / O=\lim _{n}(F / O)_{n}$. 
The homotopy of $F / O$ is recoverable from the exact sequence

$$
\cdots \rightarrow \pi_{n}(0) \stackrel{J}{\rightarrow} \pi_{n}^{S} \rightarrow \pi_{n}(F / O) \rightarrow \pi_{n-1}(0) \rightarrow \cdots,
$$

where $J$ is the Hopf-Whitehead $J$-homomorphism, and $\pi_{n}^{S}$ denotes the $n$th stable homotopy group of spheres (compare Toda [118]). This sequence is useful because the results of Kervaire and Milnor [56] lead to the following conclusion.

TheOREM 6.1 (Kervaire and Milnor, unpublished sequel to [56]; see also Levine [57]). For $n>3$ there is a long exact sequence

$$
\cdots \stackrel{\Delta}{\rightarrow} P_{n+1} \stackrel{\pi}{\rightarrow} \Theta_{n} \rightarrow \pi_{n}(F / \mathrm{O}) \stackrel{\sigma}{\rightarrow} P_{n} \rightarrow \cdots,
$$

where $P_{n}=\mathbf{Z}$ if $n \equiv 0$ (4), $\mathbf{Z}_{2}$ if $n \equiv 2$ (4), 0 if $n$ is odd. The map $\sigma_{n}$ maps onto a subgroup of finite index if $n \equiv 0 \bmod 4$.

This result has the following basic consequence.

THEOREM 6.2. The groups $\Theta_{n}, n \geqslant 4$, are finite.

The codomain of $\pi$ is given by homotopy theory, and the domain of $\Delta$ is essentially given by surgery theory. We would like a sequence similar to 6.1 for the study of $\Theta^{G}(\varphi)$. Unfortunately, this problem seems out of reach in complete generality. Therefore we shall specialize to the case $G=\mathbf{Z}_{p}$.

The results of Browder-Petrie [17], Rothenberg-Sondow [85], and others yield the following conclusions on the computation of $\Theta^{G}(\varphi)$ when $G=\mathbf{Z}_{p}$.

TheOREM 6.3. Let $\varphi$ be as usual, and let $k$ be the dimension of the fixed point set; assume $\operatorname{dim} \varphi \geqslant 6$. Then there are long exact sequences of abelian groups

$$
\begin{aligned}
\cdots & \rightarrow P_{k+1}(\varphi) \rightarrow \Theta^{G}(\varphi) \rightarrow \bigoplus_{\Theta_{k}}^{\pi_{k}(H(\varphi))} \rightarrow P_{k}(\varphi) \rightarrow \cdots, \\
& \rightarrow L_{k+1}^{k}(G) \rightarrow P_{k}(\varphi) \rightarrow \underset{\pi_{k}(F / O)}{\left.\bigoplus^{k} X(\varphi), F / O\right]} \rightarrow L_{k}^{h}(G) \cdots,
\end{aligned}
$$

where the spaces $X(\varphi)$ and $H(\varphi)$ are explicitly constructed in terms of $\varphi$, the group $\Theta_{k}$ is the Kervaire-Milnor group, $[A, B]$ represents homotopy classes of maps, and $L_{*}^{h}(G)$ denote the homotopy surgery obstruction groups (see Wall [120, Chapter 17.D]).

The precise descriptions of $X(\varphi)$ and $H(\varphi)$ are fairly elementary, but the details would require a lengthy digression. For further information see [ 90 or 93].

Since all the homotopy groups in 6.3 are finitely computable, the groups $\Theta^{G}(\varphi)$ are also computable in some sense. For example, it follows immediately that the groups $\Theta^{G}(\varphi)$ are finitely generated.

Motivated by Theorem 6.2, it is reasonable to ask for the dimension of $\Theta^{G}(\varphi) \otimes \mathbf{Q}$. This can be done in a relatively direct fashion; the groups in the 
exact sequences of 6.3 simplify dramatically when one tensors them with the rationals. In contrast with [56], the groups $\Theta^{G}(\varphi) \otimes \mathbf{Q}$ are often nonzero.

EXAMPLE. Let $\Omega_{*}^{G}$ be the group of oriented $G$-bordism classes of oriented smooth $G$-manifolds (compare Conner-Floyd [23] or Stong [113]). Then there is a natural map $\alpha: \Theta^{G}(\varphi) \rightarrow \Omega_{n}^{G}$ which sends a homotopy linear $\varphi$-sphere to its oriented $G$-bordism class. An elementary argument shows that $\alpha$ is a homomorphism. The results of J. Ewing [39] then yield the following conclusion (compare [92, Theorem 6.3(ii), pp. 33-35]):

THEOREM 6.4. Let $p \equiv 7 \bmod 8$. Then for suitable choices of $\varphi$ the map $\alpha \otimes \mathbf{Q}$ is nonzero.

Therefore in some cases homotopy linear $\varphi$-spheres give rise to highly nontrivial oriented bordism classes. By a result of Connell-Montgomery-Yang [22], the spheres in $\Theta^{G}(\varphi)$ are $G$-equivariantly homeomorphic to the linear sphere. From this and the proof of 6.4 one sees that certain rational G-characteristic numbers are not G-homeomorphism invariants. This contrasts sharply with the topological invariance of rational Pontrjagin classes (Novikov [68]).

A large number of computations for the groups $\Theta^{G}(\varphi)$ have been completed. It is beyond the scope of this article to list these in detail. Here is a very brief and incomplete list of references: Browder-Petrie [17], Rothenberg [83], and the author (e.g., [93]; several further references are listed in [93]).

Actions of more general groups. Much is known in the semifree case, for Theorem 6.3 generalizes completely. These ideas extend to a slightly wider class of actions known as ultrasemifree actions (compare [94]). This class includes all actions for which the set of isotropy groups $\left\{G_{x} \mid x \in X\right\}^{3}$ is linearly ordered by inclusion. Since the subgroups of $\mathbf{Z}_{p^{r}}$ are linearly ordered by inclusion, all actions of $\mathbf{Z}_{p^{r}}$ are ultrasemifree.

For more general actions, results thus far are quite limited. The case $G=\left(\mathbf{Z}_{p}\right)^{r}$ has been considered in unpublished work of the author [105]. It seems very likely that the exact equivariant surgery sequence of $K . H$. Dovermann and M. Rothenberg [35] should yield significant new insights in a large number of cases.

Such results would be especially valuable for actions of $p$-groups on $S^{n}$. As we have already noted, Smith theory implies that all such actions satisfy a weak analog of homotopy linearity - namely, the fixed points sets of subgroups are all homology spheres, (see Dotzel and Hamrick [32] for further analogies with linear representations). As in the case $G=\mathbf{Z}_{p}$, a good knowledge of the semilinear case, plus fixed point information as in $§ 4$, should lead to a fairly complete analysis of all smooth actions of finite $p$-groups on $S^{n}$ with nonempty fixed point sets.

7. Uniqueness of linear approximations. It is not clear exactly when the uniqueness question for linear approximations first arose, but in any case the problem was posed explicitly in a 1960 article by P. A. Smith [112]; to be

\footnotetext{
${ }^{3}$ By definition, if $x \in X$ and $G$ acts on $X$, then $G_{x}$ is the set of all $g \in G$ such that $g x=x$. It is a closed subgroup.
} 
specific, he formulated the question in terms of actions with two fixed points. Of course, since the fixed set of a $p$-group action is connected or two points ([12] once again) and the linear approximations are constant in the connected case, the two-fixed-point case is the only nontrivial one for $p$-groups.

As mentioned in Theorem 2.8, if $G=\mathbf{Z}_{p}$ acts with two fixed points $x$ and $y$ then the representations $\rho(x), \rho(y)$ are equivalent by the Atiyah-Bott fixed point formula. In fact, one can generalize 2.8 to arbitrary $p$-groups provided $p$ is odd.

THEOREM 7.1 (C. SÁNCHEZ [86]). Let $p$ be odd, and let $G$ be a p-group acting smoothly on $S^{n}$. Let $x, y$ be fixed points of the action. Then the representations $\rho(x), \rho(y)$ are linearly equivalent.

If $p=2$ the situation is far more complicated. There are two important features.

(7.2). There are numerous sufficient conditions under which $\rho(x)$ and $\rho(y)$ are linearly equivalent.

For example, if the fixed set is precisely $\{x, y\}$ and $G$ acts freely off $\{x, y\}$, then this was observed by Milnor (see [7]). The result of Sánchez extends to actions of $\mathbf{Z}_{2^{r}}$ if the lattice of isotropy subgroups $\left\{1 \subseteq H_{1} \subseteq H_{2} \subseteq \cdots \subseteq \mathbf{Z}_{2^{r}}\right\}$ satisfies $\left|H_{i+1} / H_{i}\right| \geqslant 4$. An entirely different approach of Bredon [11] implies that the difference between $\rho(x)$ and $\rho(y)$ satisfies a strong divisibility condition in the representation ring $\operatorname{RO}(G)$. Here is a specific application of [11]: If $\rho(x)=\alpha(x)+\beta, \rho(y)=\alpha(y)+\beta$, and $\operatorname{dim} \alpha(x)=\operatorname{dim} \alpha(y) \leqslant 4$, while $\operatorname{dim} \beta>4$, then $\alpha(x)$ and $\alpha(y)$ are equivalent.

On the other hand, a number of subsequent results imply that the representations $\rho(x), \rho(y)$ need not be equivalent:

(7.3) If $G=\mathbf{Z}_{2}, r \geqslant 2$, then there are numerous examples of smooth $G$-actions on homotopy $n$-spheres $\Sigma^{n}$ with $\rho(x)$ and $\rho(y)$ inequivalent.

Note. Probably one can replace $\Sigma^{n}$ by the standard sphere $S^{n}$.

Examples along these lines are due to Cappell and Shaneson [19], Siegel [111], Petrie [78-79], Dovermann [33], Suh [114] and Cho [21].

The Cappell-Shaneson examples are particularly easy to describe, so we shall give one of their pairs explicitly. If $T$ denotes the generator of $\mathbf{Z}_{4^{r}}$, then one can take $\rho(x), \rho(y)$ to be the 9-dimensional representations

$$
T^{j} \mapsto\left(\begin{array}{cc}
4 \operatorname{Rot}(\pi j / 2 r) & 0 \\
0 & (-1)^{j}
\end{array}\right),\left(\begin{array}{cc}
4 \operatorname{Rot}(\pi(2 r-j) / 2 r) & 0 \\
0 & (-1)^{j}
\end{array}\right),
$$

where $4 \operatorname{Rot}(\theta)$ means a block sum of four copies of the $2 \times 2$ rotation matrix

$$
\left(\begin{array}{rr}
\cos \theta & -\sin \theta \\
\sin \theta & \cos \theta
\end{array}\right)
$$

Results for more general groups.

1. Petrie's original results. Before the results discussed in (7.3) were proved, Petrie had considered the problem from a much different viewpoint and obtained the following result:

(7.4) (see Petrie [76, 80]). Let $G$ be an odd order abelian group that is "sufficiently large" (at least four noncyclic Sylow subgroups). Then there is a 
smooth G-action on some $S^{n}$ such that the fixed point set $F$ is finite with at least two elements, and for $x, y \in F$ the representations $\rho(x), \rho(y)$ are linearly inequivalent.

2. Actions on disks with isolated fixed points. One can ask similar questions on linear approximations to smooth actions on $D^{n}$ or $\mathbf{R}^{n}$. Results of A. Edmonds and $\mathbf{R}$. Lee [36] yield many actions on $\mathbf{R}^{n}$ with inequivalent linear approximations. On the other hand, results of Petrie [81], and Y. D. Tsai [119] yield many $G$-actions on disks with isolated fixed points and inequivalent approximations at each such point. One can construct equivariant doubles

$$
S=D \times\{0\} \cup_{\partial D} D \times\{1\} \quad(=\partial(D \times[0,1]))
$$

(compare Munkres [67] in the nonequivariant case) to obtain additional examples of smooth actions on $S^{n}$ with isolated fixed points and inequivalent linear approximations.

3. Actions with inequivalent approximations and positive-dimensional fixed point sets. Examples of this type are due to A. Assadi [2] and K. Pawalowski $[72,73]$. In [2, §IV.6, pp. 88-89] Assadi describes the proof of the following result:

THEOREM 7.5. Let $p$ and $q$ be distinct primes. Then there is a smooth $\mathbf{Z}_{p^{q}}$ action on some $S^{n}$ such that the fixed point set is two circles and the linear approximations on these components are inequivalent.

This is an application of the machinery in [ 2 and 69] in the spirit of the work of Edmonds and Lee [36]. The work of Pawalowski [72, 73] yields slightly different classes of examples. In [73] Pawalowski proves the following result.

THEOREM 7.6. Let $F_{1}, \ldots, F_{m}$ be a collection of closed, weakly almost complex $k$-manifolds whose Euler characteristics satisfy $\Sigma \chi\left(F_{j}\right)=1$. Then there is a smooth action of some finite cyclic group $G$ on some $D^{n}(n>k)$ such that:

(i) the fixed set of $G$ is diffeomorphic to the disjoint union of the $F_{j}$ 's;

(ii) the local representations $\rho_{j}$ are pairwise inequivalent.

As in the preceding discussion, one can double these actions to obtain actions on $S^{n}$.

In [73] Pawalowski constructs $G$-actions on $D^{n}$ where the fixed point set has components of different dimensions. As before, one can double the actions on disks to obtain similar actions on spheres.

We claim that the linear approximations are inequivalent for points in components of different dimensions. It is routine to check that if $x$ is a fixed point and $F_{x}$ is its component, then $\operatorname{dim} F_{x}$ equals the dimension of the subspace of $\mathbf{R}^{n}$ fixed by $G$ via $\rho(x)$. Consequently, if $\operatorname{dim} F_{x} \neq \operatorname{dim} F_{y}$, then $\rho(x)$ and $\rho(y)$ cannot possibly be linearly inequivalent.

Additional examples of this sort would be a very worthwhile addition to our knowledge.

4. Actions of odd order cyclic groups. Finally, Dovermann and Petrie have used the methods of [80] and some further ideas to prove the following result [34]:

(7.7) Let $G$ be an odd order cyclic group, and suppose that (i) no square divides the order of $G$, and (ii) the order of $G$ has a sufficiently large number of prime 
divisors. Then there is a smooth G-action on some $S^{n}$ with two fixed points $x, y$ and $\rho(x)$ inequivalent to $\rho(y)$.

Many questions remain to be solved. With the techniques currently available, and a few more insights, it might be possible to find $G$-actions with nonunique linear approximations for large families of highly nonabelian finite groups. On the other hand, for even order cyclic groups our knowledge of the possible pairs $(\rho(x), \rho(y))$ is still incomplete in many important respects. Specific questions are discussed in $[109, \S 7]$.

8. Final remarks. In assessing our current knowledge of nonlinear smooth actions on spheres, it may be helpful to give a vague analogy involving linear representations. It seems to me that present knowledge in the nonlinear case corresponds roughly to representation theory through the level of Schur's lemma. We already know a significant amount, especially for certain abelian groups, and existing techniques will certainly add to our knowledge. However, much remains to be discovered, and a more complete understanding will almost certainly include some concepts and insights that we cannot presently anticipate.

\section{REFERENCES}

1. J. F. Adams, Graeme Segal's Burnside ring conjecture, Bull. Amer. Math. Soc. (N.S.) 6 (1982), 201-210.

2. A. Assadi, Finite group actions on simply connected manifolds and $C W$ complexes, Mem. Amer. Math. Soc. No. 257 (1982).

2A. Extension of finite group actions from submanifolds of a disk, Conf. Current Trends in Algebraic Topology (Univ. of Western Ontario, London, 1981), Canad. Math. Soc. Conf. Proc., Vol. 2, Part 2, 1982, pp. 45-66.

3. A. Assadi and W. Browder, On the existence and classification of extensions of actions of finite groups on submanifolds of disks and spheres, Trans. Amer. Math. Soc (to appear).

4. M. F. Atiyah and R. Bott, A Lefschetz fixed point formula for elliptic complexes. II: Applications, Ann. of Math. (2) 88 (1968), 451-491.

5. M. F. Atiyah and I. M. Singer, The index of elliptic operators. III, Ann. of Math. (2) 87 (1968), 546-604.

6. R. H. Bing, A homeomorphism between the 3-sphere and the sum of two solid horned spheres, Ann. of Math. (2) 56 (1952), 354-362.

7. S. Bochner, Compact groups of differentiable transformations, Ann. of Math. (2) 46 (1945), 372-381.

8. A. Borel (Ed.), Seminar on transformation groups, Ann. of Math. Stud. No. 46., Princeton Univ. Press, Princeton, N. J., 1960.

9. G. Bredon, Exotic actions on spheres, Proc. Conf. Transformation Groups (New Orleans, 1967), Springer-Verlag, 1968, pp. 47-76.

10.,$A \pi_{*}$-module structure for $\Theta_{*}$ and applications to transformation groups, Ann. of Math. (2) 86 (1967), 434-448.

11. Representations at fixed points of smooth actions of compact groups, Ann. of Math. (2) 89 (1969), 515-532.

12. In I I I

13. Regular $O(n)$-manifolds, suspension of knots, and knot periodicity, Bull. Amer. Math. Soc. 79 (1973), 87-91.

14. __ Biaxial actions of classical groups, unpublished manuscript, Rutgers Univ., 1973.

15. Th. Bröcker and K. Jänich, Introduction to differential topology (translated by C. B. and M. J. Thomas), Cambridge Univ. Press, New York, 1982.

16. W. Browder, Surgery and the theory of differentiable transformation groups, Proc. Conf. Transformation Groups (New Orleans, 1967), Springer, New York, 1968, pp. 1-46. 
17. W. Browder and T. Petrie, Diffeomorphisms of manifolds and semifree actions on homotopy spheres, Bull. Amer. Math. Soc. 77 (1971), 160-163.

18. S. Cappell and J. Shaneson, Nonlinear similarity, Ann. of Math. (2) 113 (1981), 315-355.

19. __ Fixed points of periodic differentiable maps, Invent. Math. 68 (1982), 1-19.

20. S. Cappell and S. Weinberger (to appear).

21. E. C. Cho, s-Smith equivalence for representations of generalized quaternion groups, $\mathrm{Ph} . \mathrm{D}$.

Thesis, Rutgers Univ., 1984; summarized in Abstracts Amer. Math. Soc. 4 (1983), 546.

22. E. H. Connell, D. Montgomery and C. T. Yang, Compact groups in RO, Ann. of Math. (2) 80 (1964), 94-103; correction, ibid. 81 (1965), 194.

23. P. E. Conner, Differentiable periodic maps, 2nd ed., Lecture Notes in Math., vol. 738, Springer-Verlag, 1979. (First Edition written jointly with E. E. Floyd.)

24. M. Davis, Multiaxial actions on manifolds, Lecture Notes in Math., vol. 643, Springer-Verlag, 1978.

25. M. Davis, W. C. Hsiang and W. Y. Hsiang, Differential actions of compact simple Lie groups on homotopy spheres and Euclidean spaces, Proc. Sympos. Pure Math., vol. 32, part 1, Amer. Math. Soc., Providence, R. I., 1978, pp. 313-324.

26. M. Davis and W. C. Hsiang, Concordance classes of regular $U(n)$ and $S p(n)$ actions on homotopy spheres, Ann. of Math. (2) 105 (1977), 325-341.

27. M. Davis, W. C. Hsiang and J. Morgan, Concordance classes of regular $O(n)$ actions on homotopy spheres, Acta Math. 144 (1980), 153-221.

28. G. de Rham, Reidemeister's torsion invariants and rotations of $S^{n}$, Differential Analysis (Bombay Colloq., 1963), Oxford Univ. Press, New York, 1964, pp. 27-36.

29. T. tom Dieck, Semi-linear group actions on spheres: Dimension functions, Algebraic Topology Aarhus 1978 (Proc. Sympos.), Lecture Notes in Math., vol. 763, Springer-Verlag, 1979, pp. 448-457.

30. __ Homotopy representations of the torus, Arch. Math. (Basel) 38 (1982), 459-469.

31. T. tom Dieck and T. Petrie, Homotopy representations of finite groups, Inst. Haute Études Sci. Publ. Math. 56 (1982), 129-170.

32. R. Dotzel and G. Hamrick, p-group actions on homology spheres, Invent. Math. 62 (1981), 437-442.

33. K. H. Dovermann, Even dimensional s-Smith equivalent representations (Proc. Topology Conf. Aarhus, 1982), Lecture Notes in Math., vol. 1051, Springer-Verlag, 1984, pp. 587-602.

34. K. H. Dovermann and T. Petrie, Smith equivalence for odd order cyclic groups (in preparation); summarized in Abstracts Amer. Math. Soc. 4 (1983), 476-477.

35. K. H. Dovermann and M. Rothenberg, An equivariant surgery sequence and equivariant diffeomorphism and homeomorphism classification, Topology Sympos. (Siegen, 1979), Lecture Notes in Math., vol. 788, Springer-Verlag, pp. 257-280; for detailed version see preprint, Purdue Univ., 1983.

36. A. Edmonds and R. Lee, Cyclic group actions on Euclidean space, Topology 14 (1975), 339-345.

37. A. Edmonds, Transformation groups and low dimensional manifolds (Proc. Conf. Group Actions, Boulder, Colo., 1983), Contemporary Math., Amer. Math. Soc. (to appear).

38. D. Erle and W. C. Hsiang, On certain unitary and symplectic actions with three orbit types, Amer. J. Math. 94 (1972), 289-308.

39. J. Ewing, Spheres as fixed point sets, Quart. J. Math. Oxford (2) 27 (1976), 445-455.

40. E. E. Floyd, Examples of fixed point sets of periodic maps, Ann. of Math. (2) 55 (1952), $167-171$.

41. M. H. Freedman, The topology of four-dimensional manifolds, J. Differential Geom. 17 (1982), 357-453.

42. C. H. Giffen, The generalized Smith conjecture, Amer. J. Math. 88 (1966), 187-198.

43. C. McA. Gordon, On the higher dimensional Smith conjecture, Proc. London Math. Soc. (3) 29 (1974), 98-110. 1976.

44. M. W. Hirsch, Differential topology, Graduate Texts in Math., vol. 33, Springer-Verlag,

45. W. C. Hsiang and W. Y. Hsiang, The degree of symmetry of homotopy spheres, Ann. of Math. (2) 89 (1969), 52-67. 
46. (1967), 705-786

Differentiable actions of compact connected classical groups. I, Amer. J. Math. 89

47.

, Differentiable actions of compact connected Lie groups. II, III, Ann. of Math.

(1970), 189-223; ibid. 99 (1974), 220-256.

48. W. Y. Hsiang, A survey of regularity theorems in differentiable compact transformation groups, Proc. Conf. Transformation Groups (New Orleans, 1967), Springer-Verlag, 1968, pp. 77-124.

49. , On generalizations of the theorem of $A$. Borel and their applications in the study of topological actions, Topology of Manifolds (Proc. Univ. Georgia Conf., 1969), Markham, Chicago, 1970, pp. 274-290.

50. On the degree of symmetry and the structure of highly symmetric manifolds, Tamkang J. Math. 2 (1971), 1-22.

51. __ Cohomology theory of topological transformation groups, Ergebnisse Math. Grenzgebiete, Bd. 85, Springer-Verlag, 1975.

52. S. Illman, Recognition of linear actions on spheres, Trans. Amer. Math. Soc. 274 (1982), 445-478.

53. L. Jones, The converse to the fixed point theorem of P. A. Smith. I, Ann. of Math. (2) 94 (1971), 52-68.

54. $\quad$ The converse to the fixed point theorem of P. A. Smith. II, Indiana Univ. Math. J. 22 (1972), 309-325; correction, ibid. 24 (1975), 1001-1003.

55. D. S. Kahn and S. B. Priddy, The transfer and stable homotopy, Math. Proc. Cambridge Philos. Soc. 83 (1978), 103-111.

56. M. Kervaire and J. Milnor, Groups of homotopy spheres, Ann. of Math. (2) 77 (1963), 504-537.

57. J. Levine, A classification of differentiable knots, Ann. of Math. (2) 82 (1965), 15-50.

58. W. H. Lin, On conjectures of Mahowald, Segal, and Sullivan, Math. Proc. Cambridge Philos. Soc. 87 (1980), 449-458.

59. W. H. Lin et al., Calculation of Lin's Ext groups, Math. Proc. Cambridge Philos. Soc. 87 (1980), 459-469.

60. P. Löffler, Homotpielineare $Z_{p}$ Operationen auf Sphären, Topology 20 (1981), 291-312.

61. T. Matumoto, On G-CW complexes and a theorem of J. H. C. Whitehead, J. Fac. Sci. Univ. Tokyo Sect. IA Math. 18 (1971), 363-374.

62. A. Meyerhoff and T. Petrie, Quasiequivalence of G-modules, Topology 15 (1976), 69-75.

63. J. Milnor, Introduction to algebraic K-theory, Ann. of Math. Stud., No. 72, Princeton Univ. Press, Princeton, N. J., 1971.

64. J. Milnor and J. Stasheff, Characteristic classes, Ann. of Math. Stud., No. 76, Princeton Univ. Press, Princeton, N. J., 1974.

65. D. Montgomery and C. T. Yang, Differentiable transformation groups on homotopy spheres, Michigan Math. J. 14 (1967), 33-46.

66. J. W. Morgan, Actions de groupes finis sur $S^{3}$ : La conjecture de P. A. Smith (d'après Thurston et Meeks-Yau), Séminaire Bourbaki, Vol. 1980/81, Lecture Notes in Math., vol. 901, Springer-Verlag, 1981, pp. 277-289. [The results summarized in this article represent the work of several mathematicians. There are plans to publish a single volume containing most of the separate contributions.]

67. J. R. Munkres, Elementary differential topology, rev. ed., Ann. of Math. Stud., No. 54, Princeton Univ. Press, Princeton, N. J., 1966.

68. S. P. Novikov, On manifolds with free abelian fundamental groups and their applications, Izv. Akad. Nauk SSSR Ser. Mat. 30 (1966), 207-246; English transl., Amer. Math. Soc. Transl (2) 71 (1968), 1-42.

69. R. Oliver, Fixed point sets of group actions on finite acyclic complexes, Comment. Math. Helv. 50 (1975), 155-177.

70. Weight systems for SO(3) actions, Ann. of Math. (2) 110 (1980), 227-241.

71. R. Oliver and T. Petrie, G-CW surgery and $K_{0}(Z[G])$, Math. Z. 179 (1982), 11-42.

72. K. Pawalowski, Fixed points of cyclic group actions on disks, Bull. Acad. Polon. Sci. Sér. Sci. Math. Astronom. Phys. 26 (1978), 1011-1015.

73. On some problems in compact transformation groups, summarized in Math. Forschungsinst. Oberwolfach Tagungsbericht 35/1982, pp. 11-12. 
74. T. Petrie, Free metacyclic group actions on homotopy spheres, Ann. of Math. (2) 94 (1971), 108-124.

75. __ Equivariant quasiequivalence, transversality, and normal cobordism, Proc. Internat. Congress Math. (Vancouver, B. C., 1974), Canad. Math. Soc., Toronto, 1975, pp. 537-541.

76. __ Three theorems in transformation groups, Algebraic Topology Aarhus 1978 (Proc. Sympos.), Lecture Notes in Math., vol. 763, Springer-Verlag, 1979, pp. 549-572.

77. __ One fixed point actions on spheres. I, Adv. in Math. 46 (1982), 3-14.

78. T. Petrie, The equivariant J. homomorphism and Smith equivalence of representations, Conf. Current Trends in Algebraic Topology (Univ. of Western Ontario, London, 1981), Canad. Math. Soc. Conf. Proc., Vol. 2, Part 2, 1982, pp. 223-234.

79. __ Smith equivalence of representations, Math. Proc. Cambridge Philos. Soc. 94 (1983), 61-99.

80. Spherical isotropy representations, Inst. Hautes Études Sci. Publ. Math. (to appear).

81. Isotropy representations of group actions on disks (in preparation).

82. D. S. Rim, Modules over finite groups, Ann. of Math. (2) 69 (1959), 700-712.

83. M. Rothenberg, Differentiable group actions on spheres, Proc. Adv. Study Inst. on Algebraic Topology (Aarhus, 1970), Various Publications Ser., Vol. 13, Mat. Inst., Aarhus Univ., 1970, pp. 455-475.

84. Torsion invariants and finite transformation groups, Proc. Sympos. Pure Math., vol. 32, part 1, Amer. Math. Soc., Providence, R. I., 1978, pp. 267-311.

85. M. Rothenberg and J. Sondow, Nonlinear smooth representations of compact Lie groups, Pacific J. Math. 84 (1980), 427-444. (A preliminary version of this paper circulated as a Univ. of Chicago preprint in 1969.)

86. C. Sánchez, Actions of groups of odd order on compact orientable manifolds, Proc. Amer. Math. Soc. 54 (1976), 445-448.

87. R. Schultz, Improved estimates for the degree of symmetry of certain homotopy spheres, Topology 10 (1971), 227-235.

88. , Semifree circle actions and the degree of symmetry of homotopy spheres, Amer. J. Math. 93 (1971), 829-839.

89. __ Semifree circle actions with twisted fixed point sets, Proc. Second Conf. Compact Transformation Groups (Univ. of Massachusetts, Amherst, 1971), Part I, Lecture Notes in Math., vol. 298, Springer-Verlag, 1972, pp. 102-116.

90. , Homotopy sphere pairs admitting semifree differentiable actions, Amer. J. Math. 96 (1974), 308-323.

91. Closed curves and circle homomorphisms in groups of diffeomorphisms, Fund. Math. 95 (1977), 141-146.

92. __ Spherelike G-manifolds with exotic equivariant tangent bundles, Studies in Algebraic Topology, Academic Press, New York, 1979, pp. 1-39.

93. _ Differentiable actions on homotopy spheres. I: Differential structure and the knot invariant, Invent. Math. 31 (1975), 105-128.

94. __ Differentiable actions on homotopy spheres. II: Ultrasemifree actions, Trans. Amer. Math. Soc. 268 (1981), 255-297.

95. __ Differentiable actions on homotopy spheres. III: Invariant subspheres and smooth suspensions, Trans. Amer. Math. Soc. 275 (1983), 729-750.

96. __ Differentiable actions on homotopy spheres. IV: Formulas for certain normal invariants (in preparation).

97. __ Differentiable actions on homotopy spheres. V: Semifree actions on exotic spheres (in preparation).

98

Differentiable actions on homotopy spheres. VI: Circle actions on exotic spheres through dimension 30 (in preparation).

99. __ Smooth actions of small groups on exotic spheres, Proc. Sympos. Pure Math., vol. 32, part 1, Amer. Math. Soc., Providence, R. I., 1978, pp. 155-161.

100. Isotopy classes of periodic diffeomorphisms of spheres, Algebraic Topology (Proc. Waterloo, 1978), Lecture Notes in Math., vol. 741, Springer-Verlag, 1979.

101. Differentiability and the P. A. Smith theorems for spheres. I: Actions of prime order groups, Conf. Current Trends in Algebraic Topology (Univ. of Western Ontario, London, 1981), Canad. Math. Soc. Conf. Proc., Vol. 2, Part 2, 1982, pp. 235-273. 
102. Differentiability and the P. A. Smith theorems for spheres. II: Actions of larger groups (in preparation).

103. __ Exotic spheres admitting smooth circle actions with codimension four stationary sets, Proc. Northwestern Homotopy Theory Conf. (Evanston, Ill., 1982), Contemporary Math., vol. 19, Amer. Math. Soc., Providence, R. I., 1983, pp. 339-368.

104. , Upper bounds for the toral symmetry of certain homotopy spheres (Proc. Topology

Conf., Aarhus, 1982), vol. 1051, Lecture Notes in Math., Springer-Verlag, 1984, pp. 645-659.

105. __ Almost isovariant homotopy smoothings of compact $G$-manifolds (in preparation).

106. Almost extremal torus actions on homotopy spheres (in preparation).

107. Homotopy invariants for elementary abelian group actions on exotic spheres (in preparation).

108. Transformation groups and exotic spheres (Proc. Conf. Group Actions, Boulder, Colo., 1983), Contemporary Math. (to appear).

109. (Editor), Problems submitted to the A.M.S. Summer Research Conference on group actions on manifolds (Proc. Conf. Group Actions, Boulder, Colo., 1983), Contemporary Math. (to appear).

110. G. Segal, Equivariant K-theory, Inst. Hautes Études Sci. Publ. Math. 34 (1968), 129-151.

111. A. Siegel, Fixed point representations of group actions on spheres, Ph.D. Thesis, Rutgers Univ., 1982.

112. P. A. Smith, New results and old problems in finite transformation groups, Bull. Amer. Math. Soc. 66 (1960), 401-415.

113. R. Stong, Complex and oriented equivariant bordism, Topology of Manifolds (Proc. Univ. of Georgia Conf., 1969), Markham, Chicago, 1970, pp. 291-316.

114. D. Y. Suh, s-Smith equivalence for finite abelian groups, Ph.D. Thesis, Rutgers Univ., 1984; summarized in Abstracts Amer. Math. Soc. 4 (1983), 548.

115. R. W. Sullivan, Differentiable actions of classical groups on spheres, Topology 9 (1970), 155-167.

116. __ Linear models for compact group actions on spheres, Topology 13 (1974), 77-87.

117. R. Swan, Periodic resolutions for finite groups, Ann. of Math. (2) 72 (1960), 267-291.

118. H. Toda, Composition methods in homotopy groups of spheres, Ann. of Math. Stud., No. 49, Princeton Univ. Press, Princeton, N. J., 1962.

119. Y. D. Tsai, Isotropy representations of nonabelian groups on disks, Ph.D. Thesis, Rutgers Univ., 1984; summarized in Abstracts Amer. Math. Soc. 4 (1983), 545.

120. C. T. C. Wall, Surgery on compact manifolds, London Math. Soc. Monographs, vol. 1, Academic Press, New York, 1970.

121. S. Weinberger, Homologically trivial group actions. I, (preprint).

122. J. A. Wolf, Spaces of constant curvature, 4th. ed., Publish or Perish, Berkeley, Calif., 1977.

Department of Mathematics, Purdue University, West Lafayette, Indiana 47907 
